\title{
Design and Interpretation of Noninferiority Trials
}

\author{
Werner Vach, MSc, $P h D^{1,2}$ and Beryl Primrose Gladstone, $M S c, P h D^{3}$ \\ ${ }^{1}$ Institute of Medical Biometry and Statistics, Medical Faculty \& Medical Center, University of Freiburg, Freiburg, Germany; ${ }^{2}$ Department of \\ Orthopaedics and Traumatology, University Hospital Basel, Basel, Switzerland; ${ }^{3}$ Division of Infectious Disease, Department of Internal Medicine I, \\ Tübingen University Hospital, Tübingen, Germany.
}

J Gen Intern Med 33(8):1216

DOI: $10.1007 / \mathrm{s} 11606-018-4503-\mathrm{x}$

(c) Society of General Internal Medicine 2018

$\mathrm{W}$ read with great interest the paper by Aberegg and colleagues ${ }^{1}$ pointing out a rather frequent pattern of noninferiority (NI) trials with a "noninferior" or "inconclusive" conclusion though the treatment effect significantly favours the active control (AC). This standard of NI trials reporting is quite unsatisfactory, even as enhancement of superiority trial methodologies are being directed at including safety in its statistical inference, in addition to efficacy.

However, we believe it points to a deeper problem in the current conduct of NI trials. As the authors point out, a potential, assumed advantage of the new treatment (NT) is the basic logical precondition for any NI trial and is to be balanced against a potential loss in efficacy. However, this balancing is taken into account only during the planning of the trial, i.e., the decision to perform a NI trial and partially in the choice of NI margin, since guidelines ${ }^{2}$ just require preservation of effects shown in establishing the active comparator; and not at the analysis and interpretation stages.

This balancing of the observed loss in efficacy with the potential advantage, which we call the advantage deficit assessment (ADA), is what is currently missing in the conduct and interpretation of NI trials. It is a simple framework similar to the benefit-risk assessment in superiority trials balancing the advantage gained against the deficit in efficacy on a twodimensional plane and can be performed from different perspectives, e.g. societal, patient, or clinician. For example, a trial (Gallwitz 2012) ${ }^{4}$ comparing Linagliptin (NT) to Glimepiride (AC) for diabetes found $0.2 \%(0.09-0.30)$ lesser mean change in glycolated haemoglobin (deficit) and $1 \%$ lower incidence of cardiovascular events (advantage). This allows the patient/ clinician to make a choice by answering the question, "Am I ready to accept $0.30 \%$ lesser reduction in the worst case for a $1 \%$ lesser probability of cardiovascular events?" If a corresponding, desirable advantage/deficit ratio can be prespecified, it is possible to analyse NI trials within a framework close to traditional superiority studies, avoiding the unsatisfactory interpretations noted by the authors. We conducted earlier a systematic review ${ }^{3}$ of reported potential advantages and observed that only $12 \%$ of the claimed advantage were not quantifiable (e.g. avoidance of antibiotic resistance) and nearly half of the claimed advantages were already assessed implying that ADA is feasible. We recommend similar approaches to be considered when developing better standards for NI trials in accordance with the authors and other researchers.

Corresponding Author: Beryl Primrose Gladstone, MSc, PhD; Division of Infectious Disease, Department of Internal Medicine ITübingen University Hospital, Tübingen, Germany (e-mail: primrose. beryl@med.uni-tuebingen.de).

\section{Compliance with ethical standards:}

Conflict of interest: The authors declare that they have no conflict of interest.

\section{REFERENCES}

1. Aberegg SK, Hersh AM, Samore MH. Empirical consequences of current recommendations for the design and interpretation of noninferiority trials. J Gen Intern Med 2017. https://doi.org/10.1007/s11606-017-4161-4

2. Food and Drug Administration. Non-inferiority clinical trials to establish effectiveness: guidance for industry. November 2016. Available at: https:// www.fda.gov/downloads/Drugs/Guidances/UCM202140.pdf. Accessed 17 October 2017.

3. Gladstone BP, Vach $\mathbf{W}$. Analyzing noninferiority trials: it is time for advantage deficit assessment - an observational study of published noninferiority trials. Open Access J Clin Trials 2015; 7:11-21

4. Gallwitz B, Rosenstock J, Rauch T, et al. 2-year efficacy and safety of linagliptin compared with glimepiride in patients with type 2 diabetes inadequately controlled on metformin: a randomised, double-blind, noninferiority trial. Lancet 2012; 380(9840):475-483

5. Bernabe DLCR, Wangge G, Knol M, et al. Phase IV non-inferiority trials and additional claims of benefit. BMC Med Res Methodol 2013; 13:70 